\title{
CS1-CAR T Therapy
}

National Cancer Institute

\section{Source}

National Cancer Institute. CS1-CART Therapy. NCI Thesaurus. Code C155800.

Treatment with CAR T cells that are directed against the CS1 surface glycoprotein on multiple myeloma cells. 